\title{
Beta Oscillations in Working Memory, Executive Control of Movement and Thought, and Sensorimotor Function
}

\author{
ㅈobert Schmidt, ${ }^{1}$ Maria Herrojo Ruiz, ${ }^{2,3}{ }^{\circledR B}$ Bjørg E. Kilavik, ${ }^{4}{ }^{\circledR}$ Mikael Lundqvist, ${ }^{5}{ }^{\circledR}$ Philip A Starr, ${ }^{6}$ \\ and Adam R. Aron ${ }^{7}$ \\ ${ }^{1}$ Department of Psychology, University of Sheffield, Sheffield, S1 2LT, UK, 2Department of Psychology, Goldsmiths University of London, London, SE14 \\ 6NW, UK, ${ }^{3}$ Center for Cognition and Decision Making, Institute for Cognitive Neuroscience, National Research University Higher School of Economics, \\ Moscow 101000, Russian Federation, ${ }^{4}$ Institut de Neurosciences de la Timone, Aix-Marseille Université, Marseille, 13005, France, ${ }^{5}$ Department of Brain and \\ Cognitive Sciences, The Picower Institute for Learning and Memory, Massachusetts Institute of Technology, Cambridge, MA 02139-4307, ${ }^{6}$ Department of \\ Neurosurgery, University of California San Francisco, San Francisco, CA 94143, and 7Department of Psychology, University of California San Diego La Jolla,
} CA 92093

Beta oscillations ( $\sim 13$ to $30 \mathrm{~Hz}$ ) have been observed during many perceptual, cognitive, and motor processes in a plethora of brain recording studies. Although the function of beta oscillations (hereafter "beta" for short) is unlikely to be explained by any single monolithic description, we here discuss several convergent findings. In prefrontal cortex (PFC), increased beta appears at the end of a trial when working memory information needs to be erased. A similar "clear-out" function might apply during the stopping of action and the stopping of long-term memory retrieval (stopping thoughts), where increased prefrontal beta is also observed. A different apparent role for beta in PFC occurs during the delay period of working memory tasks: it might serve to maintain the current contents and/or to prevent interference from distraction. We confront the challenge of relating these observations to the large literature on beta recorded from sensorimotor cortex. Potentially, the clear-out of working memory in PFC has its counterpart in the postmovement clear-out of the motor plan in sensorimotor cortex. However, recent studies support alternative interpretations. In addition, we flag emerging research on different frequencies of beta and the relationship between beta and single-neuron spiking. We also discuss where beta might be generated: basal ganglia, cortex, or both. We end by considering the clinical implications for adaptive deep-brain stimulation.

\section{Introduction}

Since the first descriptions of sensorimotor rhythms (Berger, 1929), many researchers have pondered the functional role of beta oscillations ( $\sim 13-30 \mathrm{~Hz}$; "beta" for short). These oscillations are often prevalent during stable postures and rare during movement, and some researchers have proposed that they indicate a brain state of "neuronal activity equilibrium" or, at a more functional level, a state of "status quo" or akinesis (Jasper and Penfield, 1949; Engel and Fries, 2010; Khanna and Carmena, 2017). These neural and functional descriptions fit well with the exaggeration of beta in Parkinson's disease, with its symptoms of rigidity and slow movement (Hammond et al., 2007). However, several experimental findings do not seem readily compatible with these ideas. This has led to proposals that sensorimotor beta

Received June 27, 2019; revised Aug. 5, 2019; accepted Aug. 7, 2019.

This work was supported by the Human Brain Project (HBP-SGA1, 720270; HBP-SGA2, 785907 to R.S.); the BrainLinks-BrainTools Cluster of Excellence funded by the German Research Foundation (DFG EXC 1086 to R.S.); the British Academy (BASG161006 to M.H.R.); the BIAL Foundation (BIAL R150510 to M.H.R.); the Agence National de la Recherche (ANR-NEUR-05-045-1 to B.E.K.); the French National Centre for Scientific Research (CNRS-PEPS to B.E.K.); the Swedish Research Council (VR 2018-04197 to M.L.); the National Institute of Mental Health (NIMH R37MH087027 to M.L.); the National Institute of Health (UH3 NS100544 and R01 NS090913 to P.A.S. and NINDS NS106822 and NIDA DA026452 to A.R.A.). We thank Sumitash Jana for help with a figure.

The authors declare no competing financial interests.

Correspondence should be addressed to Robert Schmidt at robert.schmidt@sheffield.ac.uk or Adam R. Aron at adamaron@ucsd.edu.

https://doi.org/10.1523/JNEUROSCl.1163-19.2019

Copyright $\odot 2019$ the authors also has a functional role in sensorimotor integration, temporal anticipation, and confidence in expectations (Kilavik et al., 2013; Torrecillos et al., 2015; Tan et al., 2016). Furthermore, beta is also observed outside of the sensorimotor system. For example, beta occurs in prefrontal cortex (PFC) during executive control of action (Swann et al., 2009; Ruiz et al., 2011; Wessel et al., 2013), working memory (Lundqvist et al., 2016; Miller et al., 2018), and preventing distraction (Hanslmayr et al., 2014; Zavala et al., 2017), and increases in the basal ganglia (BG) in relation to sensory cues (Leventhal et al., 2012) and the encoding of sequence boundaries (Herrojo Ruiz et al., 2014). In this review, we address similarities across studies, aiming toward the larger goal of integrating these observations under a common rubric for beta.

Beta is observed using scalp EEG, MEG, intracranial electrocorticography, and local field potentials (LFPs). Although most studies have averaged beta power across trials (producing so-called event-related beta synchronizations, or desynchronizations, compared with a baseline period), recent studies have focused on beta "bursts" in single trials (Leventhal et al., 2012; Feingold et al., 2015; Lundqvist et al., 2016; Shin et al., 2017; Tinkhauser et al., 2017). The analysis of bursts reveals a rich dynamics of timing, duration, and other features. Below, we discuss results from averaged power and also from single-trial analysis, including bursts.

We start this review by considering the role of beta in PFC, both in retaining and clearing working memory. We then draw a 


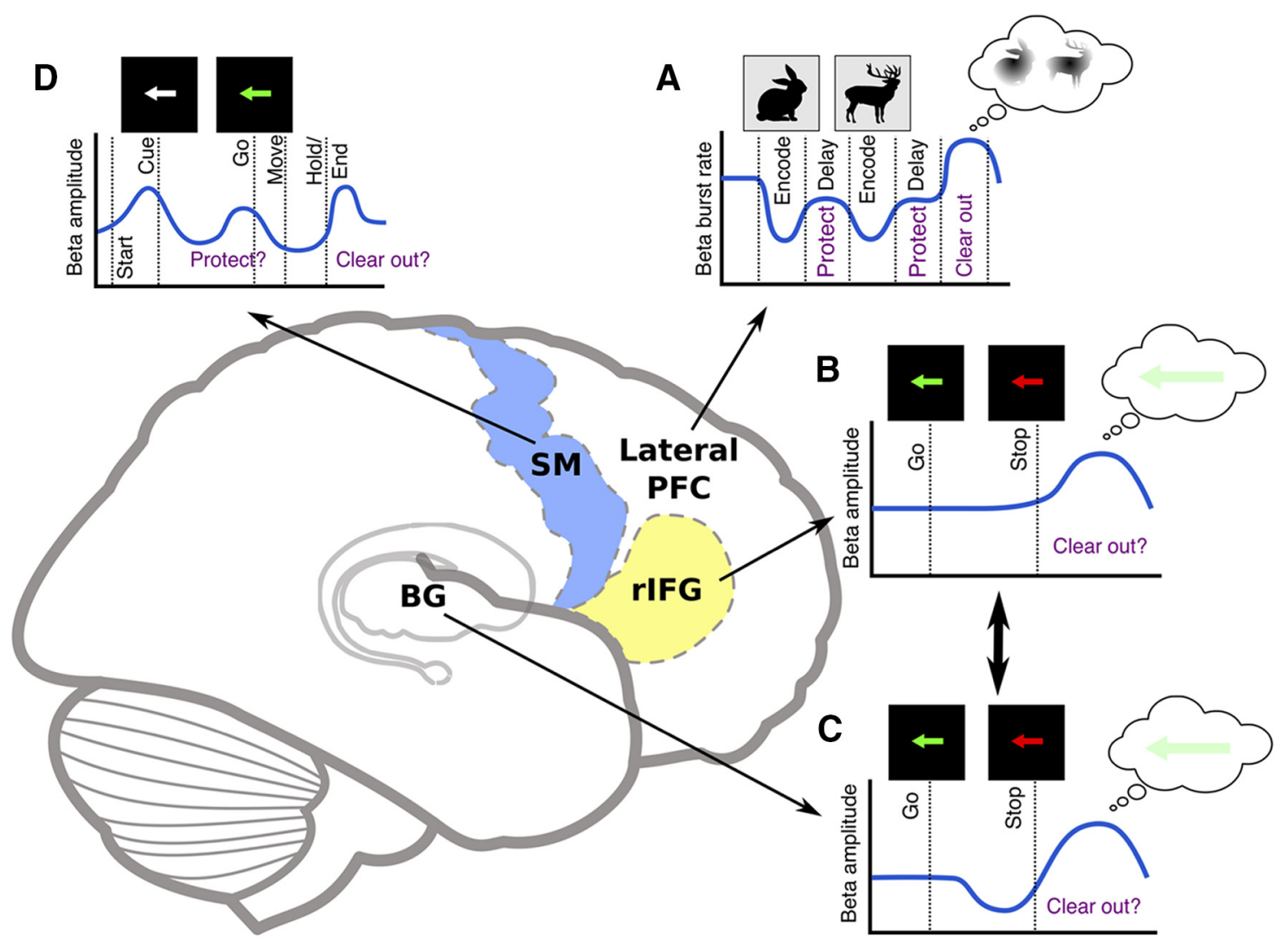

Figure 1. Schematic illustration of how beta is recruited for different tasks in different brain regions. $A$, In lateral PFC of monkeys, a working memory task required encoding two objects, then a test (Lundqvist et al., 2018). Beta decreased during the first encoding, then increased during the delay, decreased during the second encoding, then increased during the delay; finally, beta increased strongly at the end of the trial. Functionally, it was proposed that the strong beta increase at the end of the trial corresponds to clear-out, whereas the moderate increase during the delay period mediates "protection" from interference. $\boldsymbol{B}$, In the stop signal task, human subjects initiate a button press to a leftward pointing arrow, and then, when it changes color, they have to try to stop. Beta power in right inferior frontal gyrus (rIFG) increases strongly above baseline during the stop (Swann et al., 2009), possibly corresponding to clear-out. C, A similar pattern is seen when recording from the subthalamic nucleus of the BG during the stop-signal task (Ray et al., 2009): there is a desynchronization (reduction of power) relative to baseline as the subject initiates movement, but a strong increase with stopping (however, note differences in the rodent; Leventhal et al., 2012). D, In sensorimotor cortex (SM), in a pre-cued motor task in monkeys, beta amplitude is high before the cue and drops temporarily following it, before increasing again toward the go signal. Beta amplitude is minimal during the movement and then increases at the end of the trial (Kilavik et al., 2012; Kilavik et al., 2013). It is currently unclear how much the prefrontal protection and clear-out notions apply to sensorimotor beta.

connection to the suppression of movement and thought. Next, we discuss how these prefrontal and BG roles of beta relate to the well described sensorimotor beta. We then consider how beta may be generated in the cortex and BG. We end by considering the clinical implications, especially for real-time adaptive brain stimulation.

\section{Prefrontal beta for controlling contents of working memory}

Although beta has been widely studied for movement, recent findings also suggest a role in cognitive functions such as working memory (Lundqvist et al., 2011, 2016, 2018). For example, recent studies recorded PFC activity in monkeys performing a delayed match-to-sample task, in which several objects had to be encoded, maintained, and tested sequentially over several seconds (Lundqvist et al., 2016). During encoding, brief gamma bursts were associated with spiking activity while beta bursts were reduced. Then, in the following delay period, beta was increased except at the very end, when information was needed again. At that point, beta was reduced and gamma increased. Because working memory tasks typically involve a motor component (a saccade) to make the choice, this beta and gamma modulation before the test could in principle be related to movement and not cognitive aspects. However, in a follow-up study, the tests and responses were dissociated (Lundqvist et al., 2018). The observed suppression patterns of beta, and the selective upregulation of spike information about the object needed for a particular test, were consistent with a role in the flexible control of working memory rather than anticipation of movement. The pattern of beta changes is shown in Figure $1 A$. Overall, beta was reduced during encoding and test epochs, intermediate during delays, and strongly elevated after the response.

We speculate that the intermediate and strong beta increases have different functional roles. The intermediate elevation of beta during the delay period relative to the low levels seen at encoding and read-out may serve to protect the current working memory contents from interference. Indeed, human studies have shown increases of prefrontal beta when subjects must filter out distractors (Zavala et al., 2017) or prevent encoding (Hanslmayr et al., 2014). In contrast, the strong level of beta at the end of the trial might reflect a "clear-out" of the working memory content. It is noteworthy that this "beta rebound" clear-out in PFC was specific to recording sites that carried working memory information during the trial (Lundqvist et al., 2018); that is, it was not merely motor related. This opens up the intriguing possibility that, in sensorimotor cortex, the so-called postmovement beta rebound could serve a similar function for motor plans (discussed below). Overall, these studies suggest that beta bursting, originating in deep layers of PFC (Bastos et al., 2018), might explain how information is 
regulated during encoding, retention, read-out, and working memory reallocation (Lundqvist et al., 2018; Miller et al., 2018).

Noninvasive human studies have also provided evidence for an inhibitory role of alpha/beta oscillations in working memory (Jokisch and Jensen, 2007; Tuladhar et al., 2007). These signals were observed primarily in sensory cortex in a lower frequency range $(8-16 \mathrm{~Hz})$ and are thought to reflect the inhibition of taskirrelevant areas. This led to speculation that these large-scale (EEG-level findings) of alpha/beta inhibition are analogous to the fine-scale beta inhibition discussed above (Miller et al., 2018). In summary, these findings suggest that beta acts as an inhibitory filter throughout cortex, predicting when and where the contents of working memory are expressed. They also suggest possible functional similarities between cognitive and motor beta.

\section{Prefrontal-basal-ganglia beta for stopping action and thought}

As described above, beta occurred at an intermediate level in PFC during the delay period of working memory tasks, possibly to protect against interference, whereas beta occurred at high levels at the end of the trial possibly related to clear-out of the working memory content. Although those data were from monkeys during various tasks requiring control over working memory, striking parallels in prefrontal beta are seen in human tasks requiring executive control over movement and thought.

Executive control over movement can be studied with the stop-signal task (Verbruggen et al., 2019). On each trial, the subject initiates a motor response; in a minority of trials, the subject has to try to stop the movement when a subsequent stop signal occurs. A critical prefrontal region for stopping is the right inferior frontal gyrus (for review, see Aron et al., 2014). Intracranial EEG showed that after the stop signal and within a few hundred milliseconds, there was an increase in right inferior frontal beta on successful stop trials (Swann et al., 2009; Wessel et al., 2013) (Fig. 1B). A similar pattern of increased beta has been shown in several scalp EEG studies (Wagner et al., 2018; Castiglione et al., 2019). The wider network for rapidly stopping action is thought to include a hyperdirect pathway from the PFC to the subthalamic nucleus (STN) of the BG (for review, see Wessel and Aron, 2017). Consistent with this, some studies of STN LFPs during stop-signal response inhibition have revealed a relative increase of beta-band power on successful stop trials, within approximately the same time frame as for the right inferior frontal gyrus (for review, see Zavala et al., 2015; Aron et al., 2016) (Fig. 1C). Further, deep-brain stimulation of the STN in patients with Parkinson's disease led to a relative increase in right frontal beta when stopping action (Swann et al., 2011). Together, these results suggest that increased frontal and subthalamic beta reflect a network signature of the stopping process, although how communication occurs is unclear. Further, because the beta increase after the stop signal is strongly above baseline, we suppose prefrontal beta during stopping is more akin to the clear-out mode rather than protecting against interference, although this remains to be established.

Stopping might extend from movement to thought, which can be studied with the think/no-think paradigm (Anderson and Green, 2001). In the first phase, participants learn cue-target word pairs such as "oil"-"pump." In the second phase, think/nothink, they are sometimes asked to stop the retrieval process. They perform trials in which they receive the reminder word from one of the studied pairs (e.g., "oil"), presented either in green (cuing them to think of the associated word) or in red (cuing them to stop retrieval), and they are probed at the end of each trial regarding whether they experienced an intrusion of the associated memory into awareness (Levy and Anderson, 2012). A recent scalp EEG study showed that, just as for movement stopping mentioned above, there was an increase in right frontal beta during no-think trials (Castiglione et al., 2019). Strikingly, this early right frontal beta effect (beginning $\sim 300 \mathrm{~ms}$ after the nothink cue) was more pronounced during no-think trials in which retrieval was successfully stopped (i.e., there was no intrusion). These results indicate that the beta increases for successful movement-stopping and no-think trials have a common function.

How could this putative prefrontal stopping system affect the retrieval of long-term memories? Above, we saw that prefrontal beta is implicated in the control of working memory contents, including clear-out. Applying this view to the processes engaged on no-think trials, we suppose that pattern completion begins for the target word via the medial temporal lobe, but this has to then trigger reinstatement in neocortex to achieve recollection, perhaps via BG (Scimeca and Badre, 2012; Chatham and Badre, 2015). The stopping process on no-think trials, reflected in increased right frontal beta, may interfere with this latter reinstatement aspect of retrieval (also see Michelmann et al., 2016), perhaps also via $\mathrm{BG}$.

A different form of stopping might be involved in the interruption of ongoing thought (rather than preventing long-term memory retrieval), for example, when an unexpected event occurs. Because unexpected events increase beta in right frontal areas (Wagner et al., 2018) and the STN (Wessel et al., 2016), it has been proposed that unexpected events recruit a frontal-STN stopping system to interrupt working memory (Wessel and Aron, 2017).

In summary, a right frontal beta increase is associated with engagement of the stopping system for movement and also for long-term memory retrieval. It also occurs with unexpected events, which can interrupt working memory. The functional role of beta in these scenarios is perhaps most compatible with clear-out. We next consider how these putative beta functions of protecting against interference and clear-out compare to beta in sensorimotor cortex.

\section{Sensorimotor beta: amplitude, frequency, and beta bursts}

Beta in sensorimotor cortex has been characterized in more detail in terms of frequency and amplitude changes than in PFC and BG. Decades of research show that sensorimotor beta increases at rest and for stable postures, is reduced during movement, and reemerges prominently after movement or even completion of imaginary movements (for review see Kilavik et al., 2013) and also even after a passive movement (Cassim et al., 2001). For example, one study showed increased beta in both pre-cue and pre-go epochs of movement tasks, with a temporary drop in beta amplitude after the cue (Kilavik et al., 2012). This post-cue amplitude drop mainly occurs for cues containing information relevant for movement planning and parallels the decreased beta burst probability in PFC during stimulus encoding in working memory tasks (Fig. 1D). However, it remains unclear whether the increased beta amplitude in pre-cue and pre-go epochs are in some way functionally analogous to the prefrontal beta described above in reflecting, for example, protection of the posture or motor plan.

The beta rebound after movement has been linked to inhibitory GABAergic activity (for review, see Kilavik et al., 2013) and has been interpreted as an implementation of resetting mechanisms that prepare the cortical networks for the execution of upcoming movements (Pfurtscheller et al., 2005). This could align well with the putative clear-out function of beta in working 
memory. Conversely, recent studies reveal a multifaceted picture. We start by considering the relationship between beta and singleunit spiking, then we show how sensorimotor beta may have different bands with different functions, and we end with new findings on how single trial burst parameters relate to different aspects of movement.

To compare beta modulations across different studies, it is important to first understand the underlying relationship between the LFP and neuronal spiking activity. Many studies have shown that sensorimotor LFP beta at least partly reflects local activity, with the spikes of inhibitory interneurons and pyramidal tract neurons locking to the phase of beta (Murthy and Fetz, 1996; Donoghue et al., 1998; Baker et al., 1999; Jackson et al., 2002; Denker et al., 2011; Canolty et al., 2012; Confais et al., 2019). However, whether there is also an intrinsic relationship between the amplitude of beta oscillations and neuronal spike rates has been controversial (Canolty et al., 2012; Rule et al., 2017). A recent study resolved this issue (Confais et al., 2019) by showing that spike rates and beta amplitude have no intrinsic correlation, but are both modulated by external factors, such as a behavioral task.

A different issue is that the term "beta" is broad and actually involves several types of oscillations in distinct frequency bands (Kopell et al., 2011). First, in parkinsonian rats, slow and fast beta seem to take different routes through cortical and BG circuits (West et al., 2018; for human evidence, see López-Azcárate et al., 2010). Second, in humans, some evidence suggests beta frequency is effector specific, with frequencies $>20 \mathrm{~Hz}$ associated with lower limbs and frequencies $<20 \mathrm{~Hz}$ with upper limbs (Pfurtscheller et al., 2000; Neuper and Pfurtscheller, 2001). Third, in the macaque monkey, two beta bands, at $\sim 20$ and $\sim 30$ $\mathrm{Hz}$, are present in motor cortical LFPs (Kilavik et al., 2012), and phase-locking analysis of neuronal spiking activity suggests that both bands have at least partly a local origin within motor cortex (Confais et al., 2019). Whereas those particular studies found similar modulations of both bands with behavioral context and movement direction, other work found that prestimulus beta frequencies $<20 \mathrm{~Hz}$ were positively correlated with reaction times, whereas higher beta frequencies $(>20 \mathrm{~Hz})$ were negatively correlated (Zhang et al., 2008; Chandrasekaran et al., 2019). One interpretation is that the lower beta band $(<20 \mathrm{~Hz})$ is "antikinetic" (Engel and Fries, 2010), whereas the higher beta band $(>20 \mathrm{~Hz})$ reflects attention and anticipation (Saleh et al., 2010; Fujioka et al., 2012; Kilavik et al., 2012, 2014).

Finally, in addition to amplitude and frequency changes in beta, the duration, distribution and onset of beta bursts influences different properties of the movement. It was suggested that changes in beta bursts before movement was related to "specifying the movement goal," whereas fewer bursts and later bursts after an error were related to "error evaluation and monitoring" (Little et al., 2018). However, those results are perhaps also compatible with a "protection of the current state" function before movement (also see Shin et al., 2017) and, after movement error, a reduced and delayed clear-out to "buy time" to learn. We note, however, that Torrecillos et al. (2015) showed reduced postmovement beta power also for errors that do not induce motor adaptation, suggesting that the reduced beta power instead reflects the saliency of the error regardless of whether the motor plan should be preserved or updated. Other recent findings are from reward-dependent motor learning (Sporn et al., 2018). That study showed that a phasic, postreward increase in the rate of long beta bursts (duration $>500 \mathrm{~ms}$ ) attenuated the update in predictions about the rewarded movement goal (also see Tan et al., 2016). Although further work is needed to integrate these new proposals for postmovement and postfeedback sensorimotor beta, these studies highlight the usefulness of analyzing features such as duration, rate, and timing of beta bursts to better understand sensorimotor function.

In summary, whereas some aspects of sensorimotor beta might be compatible with protection of motor contents or posture and with clear-out, the picture is complicated. Recent insights into sensorimotor beta suggest that: (1) an intrinsic relationship between beta in the LFP and spikes is only present for phase-locking, not amplitude correlations; (2) there are multiple beta bands at different frequencies and these might relate to different limbs, beta-frequency changes within trials, and possibly different functional roles for beta (akinetic, attention, sensorimotor integration, and updating motor predictions); and (3) the parameters of beta bursts, such as the duration, distribution, and timing onset, relate to motor performance and learning in quite complex ways that we are just beginning to probe.

\section{Mechanisms of generating beta: BG and cortex}

Executive control, as exemplified in the context of stopping movement described above, uses beta in the cortex as well as in the BG. We start this section by considering how beta might be generated in $\mathrm{BG}$.

The BG are composed of the striatum, the globus pallidus interna (GPi) and externa (GPe), the STN, and the substantia nigra. Beta is present in all subregions of the $\mathrm{BG}$ and is modulated during the processing of sensory cues and motor signals (Leventhal et al., 2012; Herrojo Ruiz et al., 2014). As in the sensorimotor cortex and PFC, BG beta occurs in healthy animals in brief bursts, and changes in beta power typically reflect changes in the probability of beta bursts.

Much evidence has implicated the STN-GPe network in the generation of beta (Hammond et al., 2007; Mallet et al., 2008). Computational modeling has demonstrated that beta can be generated in the STN-GPe network when the inhibitory input to GPe or the excitatory input to STN is increased (Kumar et al., 2011). Changes in the inhibitory input to GPe (from striatal medium spiny neurons) also occur in awake behaving animals during movement (Cui et al., 2013). Furthermore, excitatory inputs to STN include cortical and subcortical areas, providing motor and sensory inputs (Parent and Hazrati, 1995). Therefore, the sensory and motor signals that are processed in the striatum, GPe, and STN might be related to the generation of beta in the STN-GPe networks.

A recent study (Mirzaei et al., 2017) tested whether this computational model for beta generation applies in awake behaving animals. This was done by first generating artificial activity patterns, mimicking single-unit activity recorded in the striatum, GPe, and STN of rats performing a cued choice task (Schmidt et al., 2013; Mallet et al., 2016). Second, these activity patterns were used as inputs to a spiking model of the STN-GPe network (Kumar et al., 2011). Intriguingly, the computational model generated transient beta, modulated by sensory and motor events in a way strikingly similar to that in rats performing the task. It even accounted for the positive correlation of beta with reaction times (Leventhal et al., 2012), providing a potential neural mechanism for the akinetic aspect of beta. More generally, the model demonstrated how brief changes in firing rate of the inputs to GPe and STN could lead to beta bursts. An open question is whether beta in the GPe-STN network is coordinated with cortical beta. For example, BG beta could potentially propagate to the cortex, or an independent generation of cortical beta could enable a "communication through coherence" between cortex and BG (Fries, 2015). 


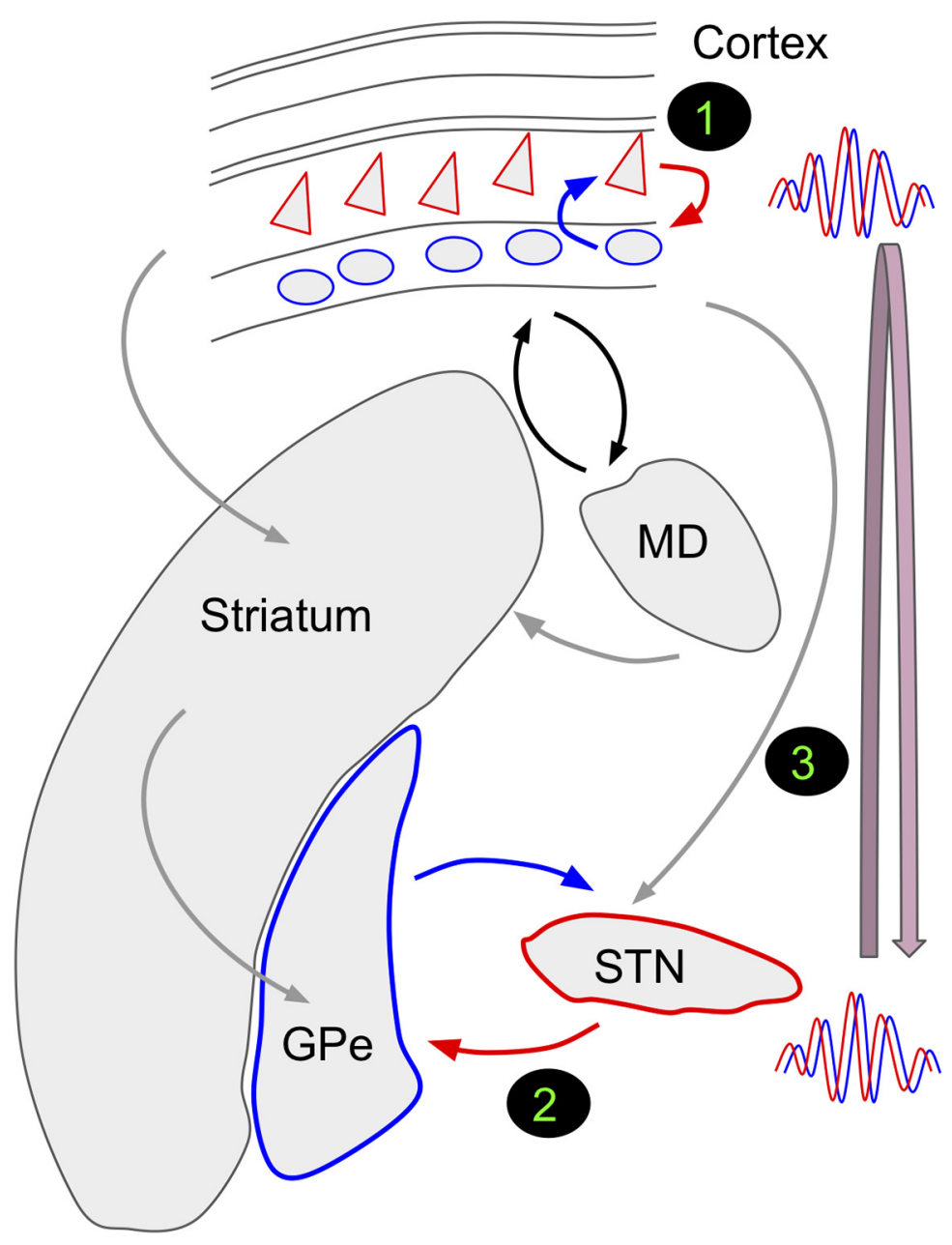

Figure 2. Schematic illustration of potential mechanisms of beta generation and interaction in thalamocortical-BG circuits. In the cortex (1), beta oscillations can be generated in deep cortical layers by interactions between pyramidal neurons (triangles) and interneurons (circles), and potentially with neurons in superficial layers (not shown). Transient beta oscillations could be triggered by excitation from the mediodorsal thalamus (MD, black arrows). In the BG, beta oscillations can be generated in the subthalamopallidal loop (2) as a result of increased striatal inhibition of GPe (e.g., due to increased input from MD) or increased excitation of STN (relevant pathways marked with gray arrows). Despite local generation in cortex and BG, the resulting beta oscillation could open a communication channel between cortical and BG circuits (3).

We now consider how cortical beta could be generated. Several lines of evidence point to cortical deep layers as a source of beta (Bollimunta et al., 2008; Buffalo et al., 2011) and possibly implicate a local circuit involving pyramidal cells and fast-spiking interneurons (via the so-called "PING" mechanism) (Lundqvist et al., 2011; Miller et al., 2018). Alternatively, interactions between excitatory and inhibitory neurons in deep and superficial layers might create beta oscillations (Sherman et al., 2016; Spitzer and Haegens, 2017) (also see Kopell et al., 2011). Strong excitation, for example from the mediodorsal thalamus (Ketz et al., 2015) to the deep layers, could lead to the generation of beta there, also in the absence of sensory inputs as required for working memory (Miller et al., 2018). Interestingly, the cortical deep layers are connected to the BG via projections to STN (RouzaireDubois and Scarnati, 1985) and via thalamocortical loops (McFarland and Haber, 2002); this might be a circuit for coordinating or propagating beta between cortex and the BG (Fig. 2).

The coordination of cortical and BG beta might orchestrate cognition and movement. One option is "top-down" communication, in which beta is generated in the cortex and then propagates to the BG. This might reflect a situation in which cortical circuits use beta to maintain stimulus information in working memory (see above) and exert control on subcortical structures to protect them against interference. By contrast, "bottom-up" communication could potentially generate beta in the STN-GPe network due to (non-oscillating) sensory and motor inputs, including ramping activity in STN (Mirzaei et al., 2017). This beta could affect reaction times and propagate through cortex via the mediodorsal thalamus. Finally, beta could be generated in the cortex and in the BG separately, perhaps relying on a shared input signal, for example, increased excitation from the mediodorsal thalamus, to both areas (Fig. 2). This might open a privileged communication channel between cortex and BG (Fries, 2015), so that spiking activity related to working memory or stopping can be processed across these circuits. Even though this remains speculative at this point, some evidence for bidirectional communication involving beta in cortical and BG circuits has been found in humans with Parkinson's disease (Lalo et al., 2008).

In summary, the interaction between STN and GPe can generate transient beta bursts observed in the BG of healthy, awake behaving rats. We do not yet know how these transient beta bursts in the BG are coordinated with cortical beta in executive function.

\section{Using the beta signature in clinical medicine}

Improving our understanding of the mechanisms and function of beta has direct clinical implications, especially for Parkinson's disease, in which there is abnormally increased beta synchronization throughout the motor network. Indeed, the aim of clinical interventions is to reduce or prevent pathological beta. Thus, understanding nonpathological beta is essential to make clinical interventions more precise and reduce potential side effects due to the removal healthy beta.

Manifestations of increased beta synchronization in Parkinson's disease include elevated resting-state beta in LFP recordings from BG nuclei (STN and GP) (Oswal et al., 2013), alteration of beta burst dynamics in the BG (Tinkhauser et al., 2017), increased beta coherence between structures of the motor network (Wang et al., 2018), and changes in the relationship between the phase of beta-frequency oscillations and the amplitude of higherfrequency oscillations in BG (López-Azcárate et al., 2010) and cortex (Swann et al., 2015; de Hemptinne et al., 2015). An important mechanism of deep-brain stimulation may be reduction of coherent oscillations between BG output (Meidahl et al., 2017) and cortex (Wang et al., 2018). Because BG beta amplitude can index the effectiveness of (levodopa) therapy (Kühn et al., 2006) or deep-brain stimulation (Kühn et al., 2008), beta amplitude recorded from BG stimulation electrodes is a promising control signal for adaptive (feedback controlled) deep-brain stimulation. 
However, caution is warranted in using STN beta for adaptive deep-brain stimulation because this signal is affected by normal movement, as well as changes in parkinsonian motor signs (Kühn et al., 2004), and the site of the maximal beta band activity within STN has connections not only within the motor system, but also with prefrontal areas that may mediate stimulation-induced adverse effects (Accolla et al., 2016). In the cortex, one effect of the parkinsonian state may be to increase beta waveform "sharpness," reflecting abnormally synchronized thalamocortical inputs (Cole et al., 2017). This raises the possibility of using waveform shape, assessed in the time domain, to index the severity of Parkinson's disease motor signs.

Much of the work on oscillatory phenomena in Parkinson's disease has been done using acute intraoperative recording in patients undergoing deep-brain stimulation surgery in the awake state, or from temporarily externalized deep-brain stimulation electrodes in the hospital. However, these recordings happen in an unnatural environment, there is a "microlesion" effect of lead insertion, there are restrictions on subject movement, and there is a limited time window for research. Helpfully, since 2013, investigators have had access to an investigational bidirectional neural interface (Activa $\mathrm{PC}+\mathrm{S}$, Medtronic) that delivers therapeutic stimulation, senses LFPs, and wirelessly streams data to an external computer (Quinn et al., 2015; Swann et al., 2018). A second-generation sensing interface, the Summit RC+S device (Medtronic), was introduced in 2018 and is the first implantable neural interface capable of continuously streaming electrophysiologic data for many hours at home. Wireless transmission of data at a distance allows full freedom of movement. Current research is using these devices to record chronic STN LFPs and primary motor cortex electrocorticography potentials in patients with Parkinson's disease, during daily motor fluctuations, and during normal activities such as hiking, driving, and sleeping (Stanslaski et al., 2018).

Chronic recordings have been used to prototype several adaptive deep-brain stimulation algorithms using primary motor cortex electrocorticography signals to deliver different levels of stimulation depending on movement state. One paradigm used motor cortex beta to increase stimulation when patients initiated movement (Herron et al., 2017). Adaptive stimulation may allow delivery of fully therapeutic deep-brain stimulation without adverse effects associated with chronic "open-loop" (unvarying) stimulation.

Apart from such research on sensorimotor motor beta in Parkinson's disease, future work may also focus on the cognitive aspects of beta. For example, one might predict that the difficulty of switching tasks in such patients with off-medication and the improvement of switching with on-medication (Cools et al., 2001) relates to changes in clear-out.

\section{Conclusions}

Recent recording studies from monkeys motivated the theory that prefrontal beta has two modes: protection and clear-out. In humans, the protective mode is perhaps compatible with studies showing increased prefrontal beta when filtering out distractors or preventing of encoding, whereas the clear-out mode may occur in relation to the stopping of movement and thoughts (canceling an incipient motor response or long-term-memory retrieval). It remains challenging to connect these possible functional roles of prefrontal beta (protection and clear-out) with the complex beta modulation observed in sensorimotor cortex during a variety of tasks. One specific avenue is to investigate possible similarities between the putative clear-out mechanism for work- ing memory content and the strong postmovement beta rebound in sensorimotor areas and to relate this to the findings on how feedback and reward are integrated to update movements. Further research on the neural mechanisms that generate beta will also help to address these open questions about the cognitive and motor functions of beta and, clinically, it will also help us better distinguish pathological from nonpathological beta.

\section{References}

Accolla EA, Herrojo Ruiz M, Horn A, Schneider GH, Schmitz-Hübsch T, Draganski B, Kühn AA (2016) Brain networks modulated by subthalamic nucleus deep-brain stimulation. Brain 139:2503-2515.

Anderson MC, Green C (2001) Suppressing unwanted memories by executive control. Nature 410:366-369.

Aron AR, Robbins TW, Poldrack RA (2014) Inhibition and the right inferior frontal cortex: one decade on. Trends Cogn Sci 18:177-185.

Aron AR, Herz DM, Brown P, Forstmann BU, Zaghloul K (2016) Frontosubthalamic circuits for control of action and cognition. J Neurosci 36:11489-11495.

Baker SN, Kilner JM, Pinches EM, Lemon RN (1999) The role of synchrony and oscillations in the motor output. Exp Brain Res 128:109-117.

Bastos AM, Loonis R, Kornblith S, Lundqvist M, Miller EK (2018) Laminar recordings in frontal cortex suggest distinct layers for maintenance and control of working memory. Proc Natl Acad Sci U S A 115:1117-1122.

Berger H (1929) Uber das elektrenkephalogramm des menschen. Archiv fur Psychiatrie und Nervenkrankheiten 87:527-570.

Bollimunta A, Chen Y, Schroeder CE, Ding M (2008) Neuronal mechanisms of cortical alpha oscillations in awake-behaving macaques. J Neurosci 28:9976-9988.

Buffalo EA, Fries P, Landman R, Buschman TJ, Desimone R (2011) Laminar differences in gamma and alpha coherence in the ventral stream. Proc Natl Acad Sci U S A 108:11262-11267.

Canolty RT, Ganguly K, Carmena JM (2012) Task-dependent changes in cross-level coupling between single neurons and oscillatory activity in multiscale networks. PLoS Comput Biol 8:e1002809.

Cassim F, Monaca C, Szurhaj W, Bourriez JL, Defebvre L, Derambure P, Guieu JD (2001) Does post-movement beta synchronization reflect an idling motor cortex? Neuroreport 12:3859-3863.

Castiglione A, Wagner J, Anderson M, Aron AR (2019) Preventing a thought from coming to mind elicits increased right frontal beta just as stopping action does. Cereb Cortex 29:2160-2172.

Chandrasekaran C, Bray IE, Shenoy KV (2019) Frequency shifts and depth dependence of premotor beta band activity during perceptual decisionmaking. J Neurosci 39:1420-1435.

Chatham CH, Badre D (2015) Multiple gates on working memory. Curr Opin Behav Sci 1:23-31.

Cole SR, van der Meij R, Peterson EJ, de Hemptinne C, Starr PA, Voytek B (2017) Nonsinusoidal beta oscillations reflect cortical pathophysiology in Parkinson's disease. J Neurosci 37:4830-4840.

Confais J, Malfait N, Brochier T, Riehle A, Kilavik BE (2019) Is there an intrinsic relationship between LFP beta oscillation amplitude and firing rate of individual neurons in monkey motor cortex? Available from: https://www.biorxiv.org/content/10.1101/586727v2.

Cools R, Barker RA, Sahakian BJ, Robbins TW (2001) Enhanced or impaired cognitive function in Parkinson's disease as a function of dopaminergic medication and task demands. Cereb Cortex 11:1136-1143.

Cui G, Jun SB, Jin X, Pham MD, Vogel SS, Lovinger DM, Costa RM (2013) Concurrent activation of striatal direct and indirect pathways during action initiation. Nature 494:238-242.

de Hemptinne C, Swann NC, Ostrem JL, Ryapolova-Webb ES, San Luciano M, Galifianakis NB, Starr PA (2015) Therapeutic deep-brain stimulation reduces cortical phase-amplitude coupling in Parkinson's disease. Nat Neurosci 18:779-786.

Denker M, Roux S, Lindén H, Diesmann M, Riehle A, Grün S (2011) The local field potential reflects surplus spike synchrony. Cereb Cortex 21: 2681-2695.

Donoghue JP, Sanes JN, Hatsopoulos NG, Gaál G (1998) Neural discharge and local field potential oscillations in primate motor cortex during voluntary movements. J Neurophysiol 79:159-173.

Engel AK, Fries P (2010) Beta-band oscillations-signalling the status quo? Curr Opin Neurobiol 20:156-165. 
Feingold J, Gibson DJ, DePasquale B, Graybiel AM (2015) Bursts of beta oscillation differentiate postperformance activity in the striatum and motor cortex of monkeys performing movement tasks. Proc Natl Acad Sci U S A 112:13687-13692.

Fries P (2015) Rhythms for cognition: communication through coherence. Neuron 88:220-235.

Fujioka T, Trainor LJ, Large EW, Ross B (2012) Internalized timing of isochronous sounds is represented in neuromagnetic beta oscillations. J Neurosci 32:1791-1802.

Hammond C, Bergman H, Brown P (2007) Pathological synchronization in Parkinson's disease: networks, models and treatments. Trends Neurosci 30:357-364.

Hanslmayr S, Matuschek J, Fellner MC (2014) Entrainment of prefrontal beta oscillations induces an endogenous echo and impairs memory formation. Curr Biol 24:904-909.

Herrojo Ruiz M, Rusconi M, Brücke C, Haynes JD, Schönecker T, Kühn AA (2014) Encoding of sequence boundaries in the subthalamic nucleus of patients with Parkinson's disease. Brain 137:2715-2730.

Herron JA, Thompson MC, Brown T, Chizeck HJ, Ojemann JG, Ko AL (2017) Cortical brain-computer interface for closed-loop deep-brain stimulation. IEEE Trans Neural Syst Rehabil Eng 25:2180-2187.

Jackson A, Spinks RL, Freeman TC, Wolpert DM, Lemon RN (2002) Rhythm generation in monkey motor cortex explored using pyramidal tract stimulation. J Physiol 541:685-699.

Jasper H, Penfield W (1949) Electrocorticograms in man: effect of voluntary move-ment upon the electrical activity of the precentral gyrus. Arch Psychiatry Zeitschr Neurol 83:163-174.

Jokisch D, Jensen O (2007) Modulation of gamma and alpha activity during a working memory task engaging the dorsal or ventral stream. J Neurosci 27:3244-3251.

Ketz NA, Jensen O, O’Reilly RC (2015) Thalamic pathways underlying prefrontal cortex-medial temporal lobe oscillatory interactions. Trends Neurosci 38:3-12.

Khanna P, Carmena JM (2017) Beta band oscillations in motor cortex reflect neural population signals that delay movement onset. Elife 6:e24573.

Kilavik BE, Ponce-Alvarez A, Trachel R, Confais J, Takerkart S, Riehle A (2012) Context-related frequency modulations of macaque motor cortical LFP beta oscillations. Cereb Cortex 22:2148-2159.

Kilavik BE, Zaepffel M, Brovelli A, MacKay WA, Riehle A (2013) The ups and downs of beta oscillations in sensorimotor cortex. Exp Neurol 245: $15-26$.

Kilavik BE, Confais J, Riehle A (2014) Signs of timing in motor cortex during movement preparation and cue anticipation. Adv Exp Med Biol 829: $121-142$.

Kopell N, Whittington MA, Kramer MA (2011) Neuronal assembly dynamics in the betal frequency range permits short-term memory. Proc Natl Acad Sci U S A 108:3779-3784.

Kühn AA, Williams D, Kupsch A, Limousin P, Hariz M, Schneider GH, Yarrow K, Brown P (2004) Event-related beta desynchronization in human subthalamic nucleus correlates with motor performance. Brain 127: 735-746.

Kühn AA, Kupsch A, Schneider GH, Brown P (2006) Reduction in subthalamic $8-35$ hz oscillatory activity correlates with clinical improvement in Parkinson's disease. Eur J Neurosci 23:1956-1960.

Kühn AA, Kempf F, Brucke C, Gaynor Doyle L, Martinez-Torres I, Pogosyan A, Trottenberg T, Kupsch A, Schneider GH, Hariz MI, Vandenberghe W, Nuttin B, Brown P (2008) High-frequency stimulation of the subthalamic nucleus suppresses oscillatory beta activity in patients with Parkinson's disease in parallel with improvement in motor performance. J Neurosci 28:6165-6173.

Kumar A, Cardanobile S, Rotter S, Aertsen A (2011) The role of inhibition in generating and controlling Parkinson's disease oscillations in the basal ganglia. Front Syst Neurosci 5:86.

Lalo E, Thobois S, Sharott A, Polo G, Mertens P, Pogosyan A, Brown P (2008) Patterns of bidirectional communication between cortex and basal ganglia during movement in patients with Parkinson disease. J Neurosci 28: 3008-3016.

Leventhal DK, Gage GJ, Schmidt R, Pettibone JR, Case AC, Berke JD (2012) Basal ganglia beta oscillations accompany cue utilization. Neuron 73:523536.

Levy BJ, Anderson MC (2012) Purging of memories from conscious awareness tracked in the human brain. J Neurosci 32:16785-16794.
Little S, Bonaiuto J, Barnes G, Bestmann S (2018) Motor cortical beta transients delay movement initiation and track errors. Available from: https://www.biorxiv.org/content/10.1101/384370v1.

López-Azcárate J, Tainta M, Rodríguez-Oroz MC, Valencia M, González R, Guridi J, Iriarte J, Obeso JA, Artieda J, Alegre M (2010) Coupling between beta and high-frequency activity in the human subthalamic nucleus may be a pathophysiological mechanism in Parkinson's disease. J Neurosci 30:6667-6677.

Lundqvist M, Herman P, Lansner A (2011) Theta and gamma power increases and alpha/beta power decreases with memory load in an attractor network model. J Cogn Neurosci 23:3008-3020.

Lundqvist M, Rose J, Herman P, Brincat SL, Buschman TJ, Miller EK (2016) Gamma and beta bursts underlie working memory. Neuron 90:152-164.

Lundqvist M, Herman P, Warden MR, Brincat SL, Miller EK (2018) Gamma and beta bursts during working memory readout suggest roles in its volitional control. Nat Commun 9:394.

Mallet N, Pogosyan A, Sharott A, Csicsvari J, Bolam JP, Brown P, Magill PJ (2008) Disrupted dopamine transmission and the emergence of exaggerated beta oscillations in subthalamic nucleus and cerebral cortex. J Neurosci 28:4795-4806.

Mallet N, Schmidt R, Leventhal D, Chen F, Amer N, Boraud T, Berke JD (2016) Arkypallidal cells send a stop signal to striatum. Neuron 89: $308-316$.

McFarland NR, Haber SN (2002) Thalamic relay nuclei of the basal ganglia form both reciprocal and nonreciprocal cortical connections, linking multiple frontal cortical areas. J Neurosci 22:8117-8132.

Meidahl AC, Tinkhauser G, Herz DM, Cagnan H, Debarros J, Brown P (2017) Adaptive deep-brain stimulation for movement disorders: the long road to clinical therapy. Mov Disord 32:810-819.

Michelmann S, Bowman H, Hanslmayr S (2016) The temporal signature of memories: identification of a general mechanism for dynamic memory replay in humans. PLoS Biol 14:e1002528.

Miller EK, Lundqvist M, Bastos AM (2018) Working memory 2.0. Neuron 100:463-475.

Mirzaei A, Kumar A, Leventhal D, Mallet N, Aertsen A, Berke J, Schmidt R (2017) Sensorimotor processing in the basal ganglia leads to transient beta oscillations during behavior. J Neurosci 37:11220-11232.

Murthy VN, Fetz EE (1996) Synchronization of neurons during local field potential oscillations in sensorimotor cortex of awake monkeys. J Neurophysiol 76:3968-3982.

Neuper C, Pfurtscheller G (2001) Evidence for distinct beta resonance frequencies in human EEG related to specific sensorimotor cortical areas. Clin Neurophysiol 112:2084-2097.

Oswal A, Brown P, Litvak V (2013) Synchronized neural oscillations and the pathophysiology of Parkinson's disease. Curr Opin Neurol 26:662-670.

Parent A, Hazrati LN (1995) Functional anatomy of the basal ganglia. II. The place of subthalamic nucleus and external pallidium in basal ganglia circuitry. Brain Res Rev 20:128-154.

Pfurtscheller G, Neuper C, Pichler-Zalaudek K, Edlinger G, Lopes da Silva FH (2000) Do brain oscillations of different frequencies indicate interaction between cortical areas in humans? Neurosci Lett 286:66-68.

Pfurtscheller G, Neuper C, Brunner C, da Silva FL (2005) Beta rebound after different types of motor imagery in man. Neurosci Lett 378:156-159.

Quinn EJ, Blumenfeld Z, Velisar A, Koop MM, Shreve LA, Trager MH, Hill BC, Kilbane C, Henderson JM, Brontë-Stewart H (2015) Beta oscillations in freely moving Parkinson's subjects are attenuated during deepbrain stimulation. Mov Disord 30:1750-1758.

Ray NJ, Jenkinson N, Brittain J, Holland P, Joint C, Nandi D, Bain PG, Yousif N, Green A, Stein JS, Aziz TZ (2009) The role of the subthalamic nucleus in response inhibition: evidence from deep-brain stimulation for Parkinson's disease. Neuropsychologia 47:2828-2834.

Rouzaire-Dubois B, Scarnati E (1985) Bilateral corticosubthalamic nucleus projections: an electrophysiological study in rats with chronic cerebral lesions. Neuroscience 15:69-79.

Ruiz MH, Strübing F, Jabusch HC, Altenmüller E (2011) EEG oscillatory patterns are associated with error prediction during music performance and are altered in musician's dystonia. Neuroimage 55:1791-1803.

Rule ME, Vargas-Irwin CE, Donoghue JP, Truccolo W (2017) Dissociation between sustained single-neuron spiking and transient beta-LFP oscillations in primate motor cortex. J Neurophysiol 117:1524-1543.

Saleh M, Reimer J, Penn R, Ojakangas CL, Hatsopoulos NG (2010) Fast and 
slow oscillations in human primary motor cortex predict oncoming behaviorally relevant cues. Neuron 65:461-471.

Schmidt R, Leventhal DK, Mallet N, Chen F, Berke JD (2013) Canceling actions involves a race between basal ganglia pathways. Nat Neurosci 16:1118-1124.

Scimeca JM, Badre D (2012) Striatal contributions to declarative memory retrieval. Neuron 75:380-392.

Sherman MA, Lee S, Law R, Haegens S, Thorn CA, Hämäläinen MS, Moore CI, Jones SR (2016) Neural mechanisms of transient neocortical beta rhythms: converging evidence from humans, computational modeling, monkeys, and mice. Proc Natl Acad Sci U S A 113:E4885-E4894.

Shin H, Law R, Tsutsui S, Moore CI, Jones SR (2017) The rate of transient beta frequency events predicts behavior across tasks and species. Elife 6:e29086.

Spitzer B, Haegens S (2017) Beyond the status quo: a role for beta oscillations in endogenous content (re)activation. eNeuro 4:ENEURO.0170-17. 2017.

Sporn S, Hein TP, Herrojo Ruiz M (2018) Bursts and variability of beta oscillations mediate the effect of anxiety on motor exploration and motor learning. Available from: https://www.biorxiv.org/content/10.1101/ $442772 \mathrm{v} 4$.

Stanslaski S, Herron J, Chouinard T, Bourget D, Isaacson B, Kremen V, Opri E, Drew W, Brinkmann BH, Gunduz A, Adamski T, Worrell GA, Denison $\mathrm{T}$ (2018) A chronically implantable neural coprocessor for investigating the treatment of neurological disorders. IEEE Trans Biomed Circuits Syst 12:1230-1245.

Swann NC, de Hemptinne C, Aron AR, Ostrem JL, Knight RT, Starr PA (2015) Elevated synchrony in Parkinson disease detected with electroencephalography. Ann Neurol 78:742-750.

Swann NC, de Hemptinne C, Miocinovic S, Qasim S, Ostrem JL, Galifianakis NB, Luciano MS, Wang SS, Ziman N, Taylor R, Starr PA (2018) Chronic multisite brain recordings from a totally implantable bidirectional neural interface: experience in 5 patients with Parkinson's disease. J Neurosurg 128:605-616.

Swann N, Tandon N, Canolty R, Ellmore TM, McEvoy LK, Dreyer S, DiSano M, Aron AR (2009) Intracranial EEG reveals a time- and frequencyspecific role for the right inferior frontal gyrus and primary motor cortex in stopping initiated responses. J Neurosci 29:12675-12685.

Swann N, Poizner H, Houser M, Gould S, Greenhouse I, Cai W, Strunk J, George J, Aron AR (2011) Deep-brain stimulation of the subthalamic nucleus alters the cortical profile of response inhibition in the beta frequency band: a scalp EEG study in Parkinson's disease. J Neurosci 31:5721-5729.
Tan H, Wade C, Brown P (2016) Post-movement beta activity in sensorimotor cortex indexes confidence in the estimations from internal models. J Neurosci 36:1516-1528.

Tinkhauser G, Pogosyan A, Tan H, Herz DM, Kühn AA, Brown P (2017) Beta burst dynamics in Parkinson's disease OFF and ON dopaminergic medication. Brain 140:2968-2981.

Torrecillos F, Alayrangues J, Kilavik BE, Malfait N (2015) Distinct modulations in sensorimotor postmovement and foreperiod beta-band activities related to error salience processing and sensorimotor adaptation. J Neurosci 35:12753-12765.

Tuladhar AM, ter Huurne N, Schoffelen JM, Maris E, Oostenveld R, Jensen O (2007) Parieto-occipital sources account for the increase in alpha activity with working memory load. Hum Brain Mapp 28:785-792.

Verbruggen F, et al. (2019) A consensus guide to capturing the ability to inhibit actions and impulsive behaviors in the stop-signal task. Elife 8:e46323.

Wagner J, Wessel JR, Ghahremani A, Aron AR (2018) Establishing a right frontal beta signature for stopping action in scalp EEG: implications for testing inhibitory control in other task contexts. J Cogn Neurosci 30:107-118.

Wang DD, de Hemptinne C, Miocinovic S, Ostrem JL, Galifianakis NB, San Luciano M, Starr PA (2018) Pallidal deep-brain stimulation disrupts pallidal beta oscillations and coherence with primary motor cortex in Parkinson's disease. J Neurosci 38:4556-4568.

Wessel JR, Aron AR (2017) On the globality of motor suppression: unexpected events and their influence on behavior and cognition. Neuron 93:259-280.

Wessel JR, Conner CR, Aron AR, Tandon N (2013) Chronometric electrical stimulation of right inferior frontal cortex increases motor braking. J Neurosci 33:19611-19619.

Wessel JR, Jenkinson N, Brittain JS, Voets SH, Aziz TZ, Aron AR (2016) Surprise disrupts cognition via a fronto-basal ganglia suppressive mechanism. Nat Commun 7:11195.

West TO, Berthouze L, Halliday DM, Litvak V, Sharott A, Magill PJ, Farmer SF (2018) Propagation of beta/gamma rhythms in the cortico-basal ganglia circuits of the parkinsonian rat. J Neurophysiol 119:1608-1628.

Zavala BA, Jang AI, Zaghloul KA (2017) Human subthalamic nucleus activity during non-motor decision making. Elife 6:e31007.

Zavala B, Zaghloul K, Brown P (2015) The subthalamic nucleus, oscillations, and conflict. Mov Disord 30:328-338.

Zhang Y, Wang X, Bressler SL, Chen Y, Ding M (2008) Prestimulus cortical activity is correlated with speed of visuomotor processing. J Cogn Neurosci 20:1915-1925. 